DOI:

10.1038/nrmicro1607

URLs

Toxoplasma gondii

http://www.ncbi.nlm.nih.gov/ entrez/query.fcgi?db=genome $\operatorname{prj} \& \mathrm{cmd}=$ RetrieveEdopt $=$ Over view\&list_uids=9535

\title{
PARASITOLOGY
}

\section{Toxo researchers spot the difference}

An estimated one billion people are infected with Toxoplasma gondii worldwide, and in Europe and North America there are just three dominant clonal lineages. Despite sharing $\sim 98 \%$ genetic identity, the three lineages - types I, II and III - differ greatly in virulence. Now, a trio of papers reveal that just a handful of genes are responsible for these differences.

In two papers in Science, Sonya Taylor, Antonio Barragan, Chunlei Su and colleagues, and Jeroen Saeij, John Boyle and colleagues used forward genetic mapping to identify the genes responsible for the strain-specific differences in virulence. In mice, type I strains are more virulent than types II and III. Taylor et al. undertook genome-wide quantitative trait loci (QTL) mapping of the F1 progeny of a genetic cross between a type I and a type III strain. They found an association between virulence and a region on chromosome VIIa, and this was narrowed down to the ROP 18 gene, which encodes the ROP18 protein. ROP18 is found in the rhoptries, specialised apical organelles that are unique to apicomplexans, and contains a conserved serine kinase domain. Taylor et al. confirmed that this protein is a functional kinase and is secreted into host cells. Importantly, transfer of the type I allele into the non-virulent type III lineage decreased the lethal dose by more than 4 logs.

Virulence is a multigenic trait and crossing the less virulent type II and type III strains generates some $\mathrm{F} 1$ progeny in which the virulence is greatly enhanced. Saeij and colleagues therefore chose to use QTL mapping in such progeny to identify loci involved in virulence. A total of five virulence loci were identified, including one on chromosome VIIa, which was found to correspond to ROP18, and one on chromosome VIIb, which corresponded to a gene encoding another rhoptry kinase, ROP16.

Rhoptry protein kinases seem to have an important role in T. gondii virulence. What are their cellular targets? This question was addressed by Jeroen Saeij, Susan Coller and colleagues in Nature. In this work, ROP16 was again identified as a key modulator of the host response, this time by a completely different approach that involved the use of microarrays to identify which genes in the F1 progeny of a cross between a type II and a type III strain had a strong effect on gene expression in human cells. Further detailed work revealed that the three clonal lineages differ in their level of activation of the STAT signalling pathway (and therefore the crucial cytokine interleukin 12 ), and that ROP16 is involved in this effect.

So, analagous to bacterial pathogens and their secretion systems, it seems that these eukaryotic pathogens can secrete protein kinases into host cells to subvert host-cell signalling pathways and that this explains many of the differences in virulence among the three dominant clonal lineages.

Sheilagh Molloy

ORIGINAL RESEARCH PAPERS Taylor, S. et al.

A secreted serine-threonine kinase determines virulence in the eukaryotic pathogen Toxoplasma gondii. Science 314, 1776-1780 (2006) | Saeij, J. P. J. et al. Polymorphic secreted kinases are key virulence factors in toxoplasmosis. Science $\mathbf{3 1 4}$, 1780-1783 (2006) | Saeij, J. P. et al. Toxoplasma co-opts host gene expression by injection of a polymorphic kinase homologue. Nature $20 \mathrm{Dec}$ 2006 (doi: 10.1038/nature05395) 